\title{
Relaxed constraints on neutrino oscillation parameters
}

\author{
Daniela Kirilova† $§$ and Mariana Panayotova $\nmid$ \\ $\dagger$ Institute of Astronomy, Sofia, Bulgaria \\ $\ddagger$ Abdus Salam ICTP, Trieste, Italy
}

\begin{abstract}
We study the cosmological constraints on active-sterile neutrino oscillations $\nu_{e} \leftrightarrow \nu_{s}$ for the case when $\nu_{s}$ is partially filled initially, i.e. $0<\delta N_{s}<$ 1. We provide numerical analysis of the cosmological production of ${ }^{4} \mathrm{He}$, in the presence of $\nu_{e} \leftrightarrow \nu_{s}$ oscillations, effective after neutrino decoupling, accounting for all known oscillations effects on cosmological nucleosynthesis. Cosmological constraints on oscillation parameters corresponding to higher than $5 \%{ }^{4} \mathrm{He}$ overproduction and different non-zero initial populations of the sterile state $\delta N_{s}<1$ are calculated. These generalized cosmological constraints corresponding to $\delta N_{s}>0$ are relaxed in comparison with the $\delta N_{s}=0$ case and the relaxation is proportional to $\delta N_{s}$.
\end{abstract}

Keywords: BBN constraints, neutrino oscillations

$\S$ To whom correspondence should be addressed (dani@astro.bas.bg) 


\section{Introduction}

Atmospheric, solar and terrestrial neutrino oscillations experiments provided evidence for neutrino oscillations. The atmospheric neutrino data analysis confirmed the $\nu_{\mu} \leftrightarrow \nu_{\tau}$ channel as the dominant atmospheric oscillation solution. The results of the terrestrial experiment KamLAND and the results of SNO salt phase prefer flavor oscillation LMA solutions to the $\nu_{e} \leftrightarrow \nu_{s}$ one for the solar neutrino problem. Active-sterile neutrino oscillations are discussed as a supplementary sub-dominant channel. Even after the confirmation of LMA solution as a dominant solution of the solar neutrino problem, the oscillation parameters are not precisely known - the completeness of LMA solution was questioned and the scope for some possible sub-dominant transitions was explored 11, 2, 3, 4, 5, 6, 7, 8. The available results of the neutrino oscillations from the solar experiments, including the later SNO ES, CC and NC data, slightly favor the existence of a small sterile oscillating sector. Thus, it is still interesting to explore the cosmological influence of sterile oscillating neutrinos.

Neutrino $\nu_{e} \leftrightarrow \nu_{s}$ oscillations affect the expansion rate and the neutrino involved processes in the early Universe and in particular, influence Big Bang Nucleosynthesis (BBN) 9, 10]. This allows to put stringent constraints on neutrino $\nu_{e} \leftrightarrow \nu_{s}$ oscillations from BBN considerations. The whole LMA and partially LOW $\nu_{e} \leftrightarrow \nu_{s}$ solar oscillation solutions and atmospheric $\nu_{e} \leftrightarrow \nu_{s}$ solution were excluded by cosmological considerations 11, 12, 13, 14, 15, 16, 17, 18, 19, 20, 21, 22, 23, 24,

Detail reviews discussing neutrino role in cosmology and neutrino masses and mixings may be found in refs. [23, 24, 25, 26].

Cosmological constraints were obtained usually assuming zero sterile neutrino state population before neutrino oscillations epoch, $\delta N_{s}=\rho_{\nu_{s}} / \rho_{\nu_{e}}^{e q}=0$, where $\rho_{\nu_{e}}^{e q}$ is the equilibrium electron neutrino energy density. However, sterile neutrinos production is predicted by different types of models, like GUT models, models with large extra dimensions, many-fold Universe models, mirror matter models, neutrino oscillations models, etc., and may be present at the onset of BBN epoch. Hence, the degree of population of $\nu_{s}$ and its initial energy spectrum distribution depends on the $\nu_{s}$ production model, and in general $\delta N_{s} \geq 0$.

The general case of initially (before oscillations) non-zero sterile neutrino population was examined and its influence on the oscillation effects at BBN epoch was discussed in reference [27]. It was found that the kinetic effect of oscillations is sensitive to the initial population of $\nu_{s}$. BBN constraints on neutrino oscillations parameters in the general case of sterile neutrino state, partially occupied initially, were discussed in reference [28] for oscillations effective after electron neutrino decoupling. The case of $3 \%{ }^{4} \mathrm{He}$ overproduction was considered. The presence of non-empty sterile neutrino $0<\delta N_{s}<0.54$ before oscillations was shown to lead to strengthening of the cosmological constraints proportional to the $\delta N_{s}$ value.

Recent studies of the systematic uncertainties of primordial ${ }^{4} \mathrm{He}$ abundance suggest as a more reliable value of ${ }^{4} \mathrm{He}$ observational uncertainty $\delta Y_{p} / Y_{p}>5 \%$. In what 
follows we derive $5.2 \%$ cosmological constraints, corresponding to $\delta Y_{p} / Y_{p}>5 \%{ }^{4} \mathrm{He}$ overproduction on $\nu_{e} \leftrightarrow \nu_{s}$ oscillation parameters for the same type of non-equilibrium neutrino oscillations. We show that in contrast to $3 \%{ }^{4}$ He constraints, in the $\delta Y_{p} / Y_{p}>$ $5 \%$ case the non-zero initial sterile population leads to a relaxation of the constraints on oscillation parameters proportional to the value of $\delta N_{s}$, and finally to their alleviation for $\delta N_{s}=1$.

In the next section, we discuss briefly the primordial ${ }^{4} \mathrm{He}$ production in the case of non-zero sterile population at the start of oscillations. In the last section we present the cosmological constraints corresponding to ${ }^{4}$ He-overproduction $\delta Y_{p} / Y_{p}>5 \%$ and different levels of initial population of the sterile state and compare them to $\delta N_{s}=0$ case.

\section{Production of primordial ${ }^{4} \mathrm{He}$ and neutrino oscillations}

\subsection{Primordial ${ }^{4} \mathrm{He}$}

${ }^{4} \mathrm{He}$ is the most abundantly produced, most precisely measured and calculated element among the primordially formed light elements. It has also a simple post-BBN chemical evolution - it is only produced in stars. Therefore, it is the preferred light element used for obtaining limits on nonstandard physics. Primordially produced ${ }^{4} \mathrm{He}$ is calculated with great precision [29, 30, 31, 32, 33] $Y_{p}=0.2485 \pm 0.0005$. The predicted 4He value is in agreement with the observational data for ${ }^{4} \mathrm{He}$ inferred from astrophysical observations 34, 35, 36, 37, 38, 39, 40].

Currently there are significantly different observational determinations of the primordial ${ }^{4} \mathrm{He}$ abundance, which point to the existence of greater systematic errors than assumed before. Izotov and Thuan have found $Y_{p} \approx 0.242 \pm 0.009$ for $82 \mathrm{HII}$ regions and $Y_{p}=0.2421 \pm 0.0021$ for 7 preferred HII regions, and $Y_{p}=0.2429 \pm 0.0009$ for an extended data set of 89 HII regions [35, 36. Olive and Skillman have re-analyzed their data and gave $Y_{p} \approx 0.245 \pm 0.013$ for all $82 \mathrm{HII}$ regions and $Y_{p}=0.2491 \pm 0.0091$ for the 7 preferred targets [37. The real uncertainty on the primordial ${ }^{4} \mathrm{He}$ abundance may be larger than previously assumed 3\%. Present determinations of primordial ${ }^{4} \mathrm{He}$ abundance from observations of extragalactic HII regions, indicate a significantly grater uncertainty for the ${ }^{4} \mathrm{He}$ mass fraction [36, 37, 39, 40] namely $d Y / Y \sim 5 \%$. ॥

Thus a derivation of cosmological constraints on oscillation parameters corresponding to about $5 \%{ }^{4} \mathrm{He}$ overproduction seems relevant.

The primordial ${ }^{4} \mathrm{He}$ abundance essentially depends on the freeze-out of nucleons, which occurs when in the process of expansion the weak processes rates $\Gamma_{w}$, governing the neutron to proton transitions, become comparable to the expansion rate $H(t)$. So, the primordially produced mass fraction of ${ }^{4} \mathrm{He} Y_{p} \sim 2(n / p)_{f} /(1+n / p)_{f}$, is a strong function of relativistic degrees of freedom at BBN epoch, which enter through $H(t)$,

\| See also the pioneer papers discussing the possibility for higher systematic errors in the determination of primordial ${ }^{4} \mathrm{He}$ abundance, which was believed to be much smaller than at present [41, 42, 43]. 
where $H(t) \sim \sqrt{G_{N} g_{e f f}} T^{2} . \S Y_{p}$ also depends on the electron neutrino characteristics, namely neutrino energy spectrum, number densities $N_{\nu}$ and the neutrino-antineutrino asymmetry, entering through $\Gamma_{w}, \Gamma_{w} \sim G_{F}^{2} E_{\nu}^{2} N_{\nu}$.

\subsection{Oscillations effects}

Non-empty initial sterile neutrino population, present at the start of oscillations, influences BBN by (i) increasing the expansion rate and (ii) suppressing the kinetic effects of $\nu_{e} \leftrightarrow \nu_{s}$ on BBN.

(i) The presence of $\delta N_{s}$ means increased effective number of neutrino species in equilibrium during $\mathrm{BBN}$, and leads to faster expansion of the Universe, $H(t) \sim g_{\text {eff }}^{1 / 2}$ causing earlier $n / p$-freezing, $T_{f} \sim\left(g_{e f f}\right)^{1 / 6}$, hence, ${ }^{4}$ He is overproduced. This dynamical effect is parametrized through $\delta Y_{d} \sim 0.013 \delta N_{s}$.

(ii) In case of oscillations between non-equilibrium sterile neutrino state $0<\delta N_{s}<1$ and electron neutrino, proceeding after $\nu$ decoupling, $\nu_{e} \leftrightarrow \nu_{s}$ oscillations lead to considerable and continuous deviations from the equilibrium $\nu_{e}$ spectrum (spectrum distortion) and production of neutrino-antineutrino asymmetry. $\|$

This effects nucleons kinetics in the pre-BBN epoch [10, 44, 27]. This kinetic effect may be parametrized in terms of additional neutrino $\delta N_{k i n}$ and will be denoted further $\delta Y_{k i n} \sim 0.013 \delta N_{k i n}$. Non-zero $\delta N_{s}$ suppresses the kinetic effect of oscillations: $\delta N_{\text {kin }}$ decreases with the increase of $\delta N_{s}$, hence $\delta N_{s}$ leads to a decrease of the overproduction of ${ }^{4} \mathrm{He}$.

For a wide range of oscillation parameters the kinetic effect of oscillations is large during the period of freezing of the nucleons and, therefore, affects BBN. This effect is strong even when there is a considerable population of the sterile neutrino state at the onset of the electron-sterile oscillations. The $\nu_{e}$ energy spectrum distortion is the greatest, if the sterile state is empty at the start of oscillations, $\delta N_{s}=0$ and decreases with the increase of the sterile state degree of population [27]. The same behavior is to be expected for the kinetic effect of oscillations.

The total effect of (i) and (ii) can be approximately described by $\delta Y_{p} \sim 0.013 \delta N$ $\delta N=\delta N_{s}+\delta N_{k i n}$, where $\delta N_{k i n}=\delta N_{k i n}^{\max }\left(1-\delta N_{s}\right)$ and $\delta N_{k i n}^{\max }$ is the kinetic oscillations effect, corresponding to $\delta N_{s}=0$. The expression presents a good approximation to the numerically calculated dependence of the kinetic effect on the initial population of $\nu_{s}$, derived in reference [27:

$\S$ Due to its strong dependence on $g_{\text {eff }}$, ${ }^{4} \mathrm{He}$ abundance was used to put constraints on the number of the relativistic particles during BBN epoch 45, 46, 47, parametrized by $\delta N_{\nu}=N_{\nu}-3$. Cosmological data (except BBN) from CMB, LSS, SN, are not very restrictive on the extra light particles 48, 49, 50, 51. || Neutrino-antineutrino asymmetry is generated during the $\nu_{e} \leftrightarrow \nu_{s}$ resonant oscillations [14. This dynamically produced asymmetry suppresses oscillations at small mixing angles. Therefore, it leads to underproduction of ${ }^{4} \mathrm{He}$ and alleviates BBN constraints on oscillation parameters compared to the case without the account of asymmetry growth. We have accounted in this work for its effect as well, however the effect is sub-dominant and will not be discussed further on. 


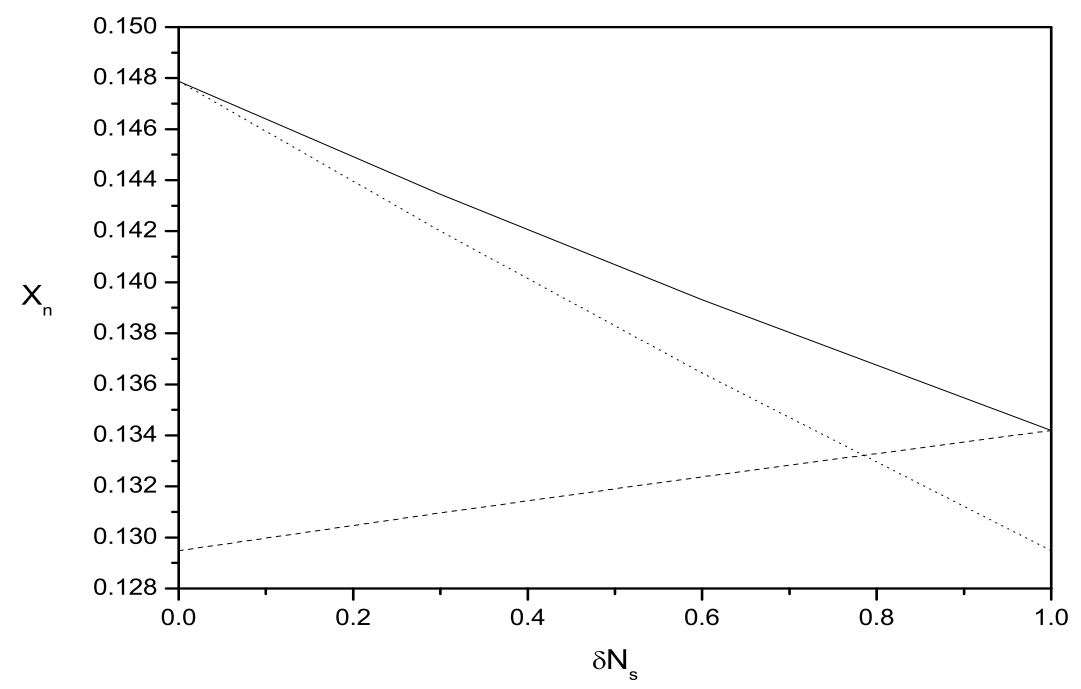

Figure 1. The solid curve presents the frozen neutron density relative to nucleons $X_{n}=n_{n}^{f} / n_{n u c}$ as a function of the sterile neutrino initial population, at $\delta m=10^{-7}$ $\mathrm{eV}^{2}$ and $\sin ^{2} 2 \theta=1$. The dotted curve presents the kinetic effect, while the dashed curve presents energy density increase effect.

There is an interesting interplay between the different effects which $\delta N_{s} \neq 0$ exerts on oscillations and on BBN with oscillations and hence, the $\delta Y_{p}$ production and the cosmological constraints on oscillations parameters for the case $\delta N_{s} \neq 0$ differ from the ones derived in references [16, 17, 52, for $\delta N_{s}=0$.

As found in [28], for $\delta Y_{p} / Y_{p}>5 \%$ corresponding to $\delta N_{k i n}^{\max }>1$, the suppression effect (ii) dominates over the dynamical effect (i) of $\delta N_{s} \neq 0$. Hence, the total effect is a decreasing function of $\delta N_{s}$, i.e. ${ }^{4} \mathrm{He}$ overproduction decreases with $\delta N_{s}$ (in comparison with the case $\delta N_{s}=0$ ) and correspondingly the BBN constraints on oscillation parameters relax. In the opposite case $\delta Y_{p} / Y_{p}<5 \%$, corresponding to $\delta N_{k i n}^{\max }<1$, the dynamical effect (i) dominates and the total effect is increasing with $\delta N_{s}$. I.e. ${ }^{4} \mathrm{He}$ overproduction increases and the BBN constraints on oscillations strengthen in comparison with the case $\delta N_{s}=0$. In the case $\delta Y_{p} / Y_{p}=5 \%$ the constraints for $\delta N_{s} \neq 0$ coincide with the ones for $\delta N_{s}=0$, due to the cancellation of the two effects (i) and (ii).

For illustration of $\delta N_{k i n}^{\max }>1$ case, which we consider further on, we present in Figure 1 the effects (i) and (ii) on ${ }^{4} \mathrm{He}$ overproduction at $\delta m=10^{-7} \mathrm{eV}^{2}$ and $\sin ^{2} 2 \theta=1$. We have studied numerically the contribution of these effects on neutrons to nucleons freezing ratio $X_{n}=n_{n}^{f} / n_{n u c}$ for different $\delta N_{s}$. The primordial yield of helium to a good approximation is expressed through $X_{n}: Y_{p} \sim X_{n} \exp \left(-t / \tau_{n}\right)$, where $\tau_{n}$ is the neutron lifetime. For the chosen set of parameters the ${ }^{4} \mathrm{He}$ overproduction decreases with the increase of $\delta N_{s}$. The suppression effect (ii) of $\delta N_{s}$ dominates and a relaxation of the cosmological constraints compared to $\delta N_{s}=0$ must be expected. 
In the next section we present and discuss the calculated cosmological constraints on oscillation parameters corresponding to higher than $5 \%{ }^{4} \mathrm{He}$ overproduction and $0<\delta N_{s}<1$.

\section{Cosmological constraints on oscillation parameters}

BBN constraints corresponding to $\delta Y_{p} / Y_{p}=3 \%$ overproduction of ${ }^{4} \mathrm{He}$ and non-zero initial population of the sterile neutrino $\delta N_{s}<0.54$ were calculated recently [28]. As far as $\delta Y_{p} / Y_{p}=3 \%$ corresponds to $\delta N_{k i n}^{\max }<1$, the constraints strengthen with the increase of the $\delta N_{s}$ value. They increase the BBN 3\% ${ }^{4} \mathrm{He}$ exclusion region for oscillation parameters corresponding to $\delta N_{s}=0$ towards smaller $\delta m^{2}$.

Having in mind the existence of a large systematic error of ${ }^{4} \mathrm{He}$ measurements, in this work we provide a numerical calculation of cosmological constraints corresponding to $\delta Y_{p} / Y_{p}>5 \%{ }^{4} \mathrm{He}$ overproduction $\left(\delta N_{k i n}^{\max }>1\right)$ and different initial degrees of sterile neutrino population in the range $0 \leq \delta N_{s}<1$. We have chosen $\delta Y_{p} / Y_{p}=5.2 \%$, i.e. a value slightly higher than the critical one $5 \%$, inorder to illustrate the possibility for relaxation of the cosmological constraints on oscillations for $\delta N_{s} \neq 0$.

Our numerical analysis has shown that cosmological constraints corresponding to $5.2 \%{ }^{4} \mathrm{He}$ overproduction relax with the increase of $\delta N_{s}$ values. Up to $\delta N_{s}=0.5$ the cosmological constraints are slightly relaxed in comparison with the case $\delta N_{s}=0$, however, for higher $\delta N_{s}$ values, the constraints relax noticeably. The reason for that is the predominance of (ii) effect (the suppression of the oscillations kinetic effects) over the dynamical effect (i) for the given uncertainty of ${ }^{4} \mathrm{He}$. All cosmological constraints corresponding to $\delta Y_{p} / Y_{p}>5 \%$ will have such behaviour, namely, they will be relaxed in comparison to the constraints for $\delta N_{s}=0$. (Vice versa, the constraints corresponding to lower than $5 \%{ }^{4} \mathrm{He}$ uncertainty will be more stringent than the ones for initially empty sterile neutrino state.)

In Figure $2 \delta Y_{p} / Y_{p}=5.2 \% \mathrm{BBN}$ constraints are presented for different values of the initial population of the sterile state, namely the lowest dashed contour corresponds to a zero population, the solid curve corresponds to $\delta N_{s}=0.5$, the dotted and the dot dashed contours to $\delta N_{s}=0.7$ and to $\delta N_{s}=0.9$, respectively.

Another interesting result is that there are considerable constraints even for a very high $\delta N_{s}$ values for that really high ${ }^{4} \mathrm{He}$ uncertainty. I.e. the constraints on neutrino mixing parameter vanish only when the sterile state is in equilibrium before oscillations, when the kinetic effect due to neutrino spectrum distortion disappears, i.e. $\delta N_{s}=1$.

\section{Conclusions}

We have studied BBN constraints on neutrino $\nu_{e} \leftrightarrow \nu_{s}$ oscillations for the specific case when the sterile neutrino is partially filled initially $0<\delta N_{s}<1$. Non-zero $\delta N_{s}$ has two-fold effect on BBN with neutrino oscillations: a dynamical effect leading to overproduction of He-4 and a kinetic effect, leading to underproduction of He-4 in 


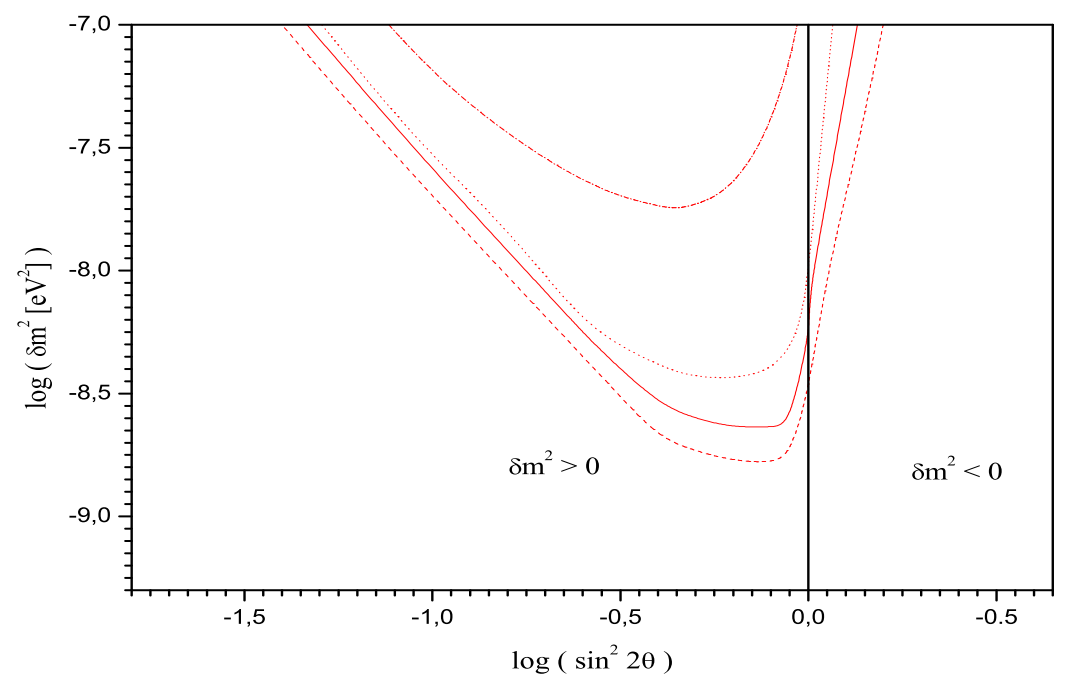

Figure 2. The dashed contour presents $\delta Y_{p} / Y_{p}=5.2 \%$ BBN constraints for $\delta N_{s}=0$, the solid curve corresponds to $\delta N_{s}=0.5$, the dotted and the dot dashed contours $\delta N_{s}=0.7$ and to $\delta N_{s}=0.9$, respectively. The resonant oscillations case corresponds to l.h.s of the figure, the non-resonant one to the r.h.s..

comparison with the case of delta $N_{s}=0$. So, depending on the interplay between these opposite effects, the cosmological constraints may be either relaxed or strengthened.

We have provided detail numerical analysis of the $\mathrm{BBN}$ production of ${ }^{4} \mathrm{He}, Y_{p}$, in the presence of $\nu_{e} \leftrightarrow \nu_{s}$ neutrino oscillations, effective after electron neutrino decoupling. We have calculated and discussed cosmological constraints on oscillation parameters, corresponding to higher than $5 \%$ uncertainty of helium-4, for non-zero initial population of the sterile state $\delta N_{s}<1$. It was found that the cosmological constraints on oscillation parameters relax with the increase of $\delta N_{s}$. The cosmological constraints for the cases $\delta N_{s} \leq 0.5$ are slightly changed in comparison to $\delta N_{s}=0$ case, however, for bigger $\delta N_{s}$ the constraints are relaxed considerably and for $\delta N_{s}=1$ they are alleviated.

Resuming the results of the works discussing $\delta N_{s}$ effect on BBN: Cosmological constraints corresponding to higher than $5 \%$ uncertainty of helium-4, relax with the increase of the initial population of the sterile state, while the constraints corresponding to lower than $5 \%$ uncertainty of helium- 4 strengthen with $\delta N_{s}$.

It is remarkable, that in case of BBN with non-equilibrium oscillations between electron and sterile neutrinos, it is possible to obtain cosmological constraints on oscillation parameters even in the case when the ${ }^{4} \mathrm{He}$ abundance is known with uncertainty greater than $5 \%$ (Actually, it is possible to derive constraints on neutrino oscillation parameters for He- 4 uncertainty up to $32 \%$ in the resonant oscillations case, and up to $14 \%$ uncertainty in the nonresonant oscillations case, as far as these are the maximal possible helium overproduction values [53]). The cosmological constraints persist while initially the sterile state is non equilibrium. When $\delta N_{s}=1$ initially, 
the kinetic effect of oscillations is zero and it is not possible to obtain constraints on oscillation parameters in the discussed BBN models with non-equilibrium electron-sterile neutrino oscillations, as well as in BBN models with equilibrium electron-sterile neutrino oscillations.

\section{Acknowledgments}

The authors thank the referee for the useful comments and suggestions. D. Kirilova appreciates the visiting position at ULB, Bruxelles and the Regular Associateship of the Abdus Salam ICTP, Trieste. This work was supported in part by Belgian Federal

Government (Federal Public Planning Service Science Policy (PPS Science Policy)).

\section{Referencing}

[1] Aliani P et al 2003 CERN-TH-2003-150; Preprint hep-ph/0309156

[2] Kang K S, Kim C S 2003 Preprint hep-ph/0306210

[3] Kang K S, Kim C S 2004 Phys. Lett. B 584 98; Preprint hep-ph/0403059

[4] Chauhan B C, Pulido J 2004 JHEP B 0406 008; Preprint hep-ph/0402194

[5] Holanda P, Smirnov A 2004 Phys. Rev. D 69 113002; Preprint hep-ph/0307266

[6] Dev S, Sanjeev K 2005a Mod. Phys. Lett. A 202083

[7] Dev S, Sanjeev K 2005b Mod. Phys. Lett. A 20 2957; Preprint hep-ph/0504237

[8] Pulido J, Chauhau B 2005 Nucl. Phys. B 14537

[9] Dolgov A D 1981 Sov. J. Nucl. Phys. B 5067

[10] Kirilova D 1988 JINR E2-88-301

[11] Barbieri R, Dolgov A 1990 Phys. Lett. B 237440

[12] Barbieri R, Dolgov A 1991 Nucl. Phys. B 349743

[13] Enqvist K et al 1992 Nucl. Phys. B 373498

[14] Kirilova D, Chizhov M 1997 Phys. Lett. B 393375

[15] Kirilova D 2004 Central Eur. J. Phys 2 467; Preprint astro-ph/0312569

[16] Kirilova D, Chizhov M 1998 Phys. Rev. D 58073004

[17] Kirilova D, Chizhov M 2000 Nucl. Phys. B 591457

[18] Dolgov A D 2001 Phys. Lett. 33700

[19] Di Bari P 2002 Phys. Rev. D 65 043509; 2003 Phys. Rev. D 67127301

[20] Cirelli M 2004 Preprint astro-ph/0410122

[21] Cirelli M et al 2005 Nucl. Phys. B 708 215; Preprint hep-ph/0403158

[22] Dolgov A D, Villante F 2004 Nucl. Phys. B 679261

[23] Dolgov A D 2002 Phys. Rept. 370333

[24] Kirilova D, Chizhov M 2001 Dubna Hot points in Astrophysics 56; Preprint astro-ph/0108341

[25] Strumia A, Vissani F 2006 Preprint hep-ph/0606054

[26] Lesgourgues J, Pastor S 2006 Phys. Rept. 429307

[27] Kirilova D 2004 Int. J. Mod. Phys. D 13 831; Preprint hep-ph/0209104 v.3

[28] Kirilova D 2004 Preprint astro-ph/0511231

[29] Lopez R E, Turner M S 1999 Phys. Rev. D 59103502

[30] Cyburt R et al 2003 Phys. Lett. B 567227

[31] Cyburt R 2004 Phys. Rev. D 70 0203505; Preprint astro-ph/0401091/v.2

[32] Cuoco A et al 2004 Int. J. Mod. Phys. A 19 4431; Preprint astro-ph/0307213

[33] Coc A et al 2004 Ap. J. $\mathbf{6 0 0} 544$

[34] Olive K et al 1997 Ap. J. 483788

[35] Izotov Yu I, Thuan T X 1998 Ap. J. 500188 
[36] Izotov Yu I, Thuan T X 2004 Ap. J. 602200

[37] Olive K, Skillman E 2004 Ap. J. 617 29; Preprint astro-ph/0405588

[38] Fields B, Sarkar S 2004 Phys. Lett. B 592 1; Preprint astro-ph/0406663

[39] Cyburt R et al 2005 Astropart. Phys. 23 313; Preprint astro-ph/0408033

[40] Fukugita M, Kawasaki M 2006 Preprint astro-ph/0603334

[41] Sasselov D, Goldwirth D 1995 Ap. J. 444 L5

[42] Luridiana V 2003 Proceedings of the XXXVIIth Rencontres de Moriond 159; Preprint astro-ph/0209177

[43] Luridiana V et al 2003 Ap. J. $\mathbf{5 9 2} 846$

[44] Kirilova D 2003 Astropart. Phys. 19409

[45] Shvartsman V 1969 Pisma Zh.Eksp.Teor.Fiz. 9 315; JETP Lett. 9184

[46] Steigman G et al 1986 Phys. Lett. B 17633

[47] Lisi E et al 1999 Phys. Rev. D 59123520

[48] Trotta R. , Hansen S. 2004 Phys. Rev. D 69023509

[49] Hannestad S, Raffeld G G 2006 Preprint astro-ph/0607101

[50] Hannestad S 2006 JCAP 0601001

[51] Cirreli M, Strumia A 2006 Preprint astro-ph/0607086

[52] Kirilova D, Chizhov M 2001 Nucl. Phys. Suppl. B 100360

[53] Kirilova D 2003 Astropart. Phys. 19409 